\title{
Potential Use of Botanicals Rich in Alkaloids for Controlling Meloidogyne incognita and Rotylenchulus reniformis Infecting Cucurbits
}

\author{
Fatma A. M. Mostafa*, A. R. Refaei*, A. E. Khalil** and Marwa M. El-Deriny** \\ *Nematology Research Unit, Agric. Zool. Dept., Fac. Agric., Mansoura Univ., Mansoura. Egypt \\ **Plant Pathol. Res. Inst., Agric. Res. Center, Giza, Egypt.
}

\begin{abstract}
Two greenhouse experiments were conducted to evaluate the nematicidal activity of certain alkaloid plants namely, periwinkle (Catharanthus roseus), datura (Datura stramonium), lupin (Lupinus albus), pomegranate (Punica granatum) and fenugreek (Trigonella foenum-graecum) against root-knot nematode Meloidogyne incognita infecting cucumber (Cucumis sativus) and reniform nematode (Rotylenchulus reniformis) infecting cantaloupe (Cucumis melo var.cantaloupensis). Dried powdered parts of such plants were screened at three rates (1,3 and $5 \mathrm{~g} /$ plant). In most treatments data indicated a positive correlation between tested rates and plant biomass. Dried leaf powder of $C$. roseus $(62.5 \%)$ as well as dried seed powder of $L$. albus ( $52.8 \%$ ), and $T$. foenum-graecum ( $43.2 \%$ ) induced remarkable improvement in total plant fresh weight of cucumber at the rate of $5 \mathrm{~g} /$ plant. However, dried leaf powder of $D$. stramonium (41.4\%) recorded a significant increase in total plant fresh weight at the rate of $3 \mathrm{~g} / \mathrm{plant}$. By all treatments of alkaloid plants root-knot nematode population whether in soil and roots, root galling and number of egg masses were significantly suppressed. Dried leaf powder of $C$. roseus at three tested rates $(71.8,71.6$ and $72.6 \%)$ performed the best and suppressed total nematode population of $M$. incognita followed by $T$. foenumgraceum at $5 \mathrm{~g} /$ plant $(70.1 \%)$. On the other hand, a slight to moderate improvement in plant growth parameters of cantaloupe infected with $R$. reniformis was indicated in pots receiving alkaloids plants. The highest percentage of increase in total plant fresh weight was noticed with dried leaf powder of $C$. roseus at three tested rates $(28.5,31.4$ and $32.6 \%)$ respectively. The best protection of cantaloupe against $R$. reniformis was recorded with dried seed powder of $T$. foenum-graecum $(57.9,65.2$ and $75.0 \%)$ and $P$. granatum $(55.4,55.9$ and $61.7 \%)$ at three tested rates, respectively which ultimately suppressed $R$. reniformis population.
\end{abstract}

Key words: Meloidogyne incognita, Rotylenchulus reniformis, cucumber, cantaloupe, alkaloid plants.

\section{Introduction}

Cucumber (Cucumis sativus) and cantaloupe (Cucumis melo var. cantaloupensis) belonging to the family cucurbitaceae are monoecious and warm- 
season plants (Amar and Lev, 2011) that performed best in fertile and well drained loamy soil. In Egypt, cucumber was produced on 49702 feddans with an average of 9.188 tons /feddan during the season of 2013/2014. Cantaloupe was produced on 71149 feddans with an average of 12.461 tons/feddan in the same season (Minis. Agric., Egypt).

Root-knot (Meloidogyne spp.) and reniform (Rotylenchulus reniformis) nematodes are considered as worldwide important pests on cucurbits causing considerable yield loss. The above-ground symptoms of these nematodes include stunting, early wilting, malformed fruits and poor yield (Dorman and Nelson, 2012). The root system infected with Meloidogyne spp. is characterized by gall formation. However, the damage to that infected with $R$. reniformis include reduced feeder roots, dead tissue and significant reduction in root growth. Nematode damage may induce some members of the cucurbit family to produce large amounts of ethylene gas which can contribute to premature ripening of fruit (Noling, 2009). Controlling such nematodes by chemical methods are quick and effective. However, deleterious effects of nematicides on environment and human as well as disruption of biological balance and high cost have created a new eco-friendly strategy for nematode management. In the nature, plants produce a number of secondary metabolites to protect themselves from various pests and pathogens. Such metabolites include alkaloids, terpenoids, glycosides and flavonoids. The botanical pesticides offer safe alternative strategy for nematode management due to their facile biodegradability and selective toxicity to target organisms (Archana and Prasad, 2014).

Different plant materials have been used by many researchers to evaluate their nematicidal properties and have been reported to be toxic to plant parasitic nematodes (Goswami and Vijaylakshmi, 1986; Zia et al., 2001; Ismail, 2014 and Umar and Ngwamdai, 2015). Alkaloids are nitrogenous compounds that possess antibacterial and antiviral activities (Luijendijk, et al., 1996; Suhad et al., 2004). Certain plant families viz. Papaveraceae (poppy) Ranunculaceae (buttercups), Solanaceae (nightshades), and Amaryllidaceae (amaryllis) are particularly rich in alkaloids. Botanical extracts that contain alkaloids were found to have ovicicidal property against Meloidogyne eggs (Adegbite, 2003). Therefore, the main objective of the present study was to evaluate the nematicidal activity of certain botanicals rich in alkaloids on Meloidogyne incognita and Rotylenchulus reniformis infecting cucumber and cantaloupe, respectively under greenhouse conditions.

\section{Materials and Methods}

Plastic pots $15 \mathrm{~cm}$-d were filled with $800 \mathrm{~g}$ sandy loamy soil (1:1) (v:v). Pots were supplemented with dried powder of selective plants rich in alkaloids i.e periwinkle (Catharanthus roseus), datura (Datura stramonium), lupin (Lupinus albus), pomegranate (Punica granatum) fenugreek (Trigonella foenum-graecum) at 
three rates $(1,3$ and $5 \mathrm{~g} / \mathrm{pot})$. Three pots were treated with the conventional nematicide oxamyl $(10 \% \mathrm{G})$ two days after nematode inoculation at the rate of $0.3 \mathrm{~g} /$ pot. However, three pots were left free of nematode infection and any treatment to serve as control. Another three pots were received nematode alone. Pots supplemented with dried alkaloid plants were maintained moist for 7 days to allow decomposition. Seeds of cucumber cv. Themin were then planted in each pot. After 15 days, plants were thinned to one seedling and inoculated with 1000 second stage juveniles (J2) of Meloidgyne incognita perpot.

Treatments were as follows:

1. Dried leaf powder of $C$. roseus (1,3 and $5 \mathrm{~g} / \mathrm{pot})$,

2. Dried leaf powder of $D$. stramonium(1, 3 and $5 \mathrm{~g} / \mathrm{pot})$,

3. Dried seed powder of $L$. albus (1, 3 and $5 \mathrm{~g} / \mathrm{pot})$,

4. Dried fruit peels powder of $P$. granatum $(1,3$ and $5 \mathrm{~g} / \mathrm{pot})$,

5. Dried seed powder of $T$. foenum-graecum (1, 3 and $5 \mathrm{~g} / \mathrm{pot})$,

6. Oxamyl,

7. Nematode alone and

8. Untreated plant.

Pots were arranged on a greenhouse bench in a randomized complete block design, with three replicates for each treatment at $22 \pm 5^{\circ} \mathrm{C}$. Pots received water as needed and maintained for 45 days after nematode inoculation. At the end of experiment plants were harvested. Data dealing with fresh shoot and root length, shoot and root weight and shoot dry weight were recorded. Plant roots were stained in 0.01 acid fuchsin in lactic acid (Byrd et al.,1983) and examined for the number of developmental stages, females, galls and egg masses. Root galling and egg masses was rated on a scale of $0-5$ where $0=$ no galls or egg masses, $1=1-2,2=$ $3-10,3=11-30,4=31-100$ and $5=$ more than 100 galls or egg masses (Taylor and Sasser, 1978). Vermiform stages of $M$. incognita were extracted from the soil using sieving and modified Baremann technique (Goodey, 1957).

The same methodology was repeated with $R$. reniformis at inoculum level 500 vermiform immature females on cantaloupe at $27 \pm 3^{\circ} \mathrm{C}$. At the end of experiment plants were harvested. Data dealing with fresh plant length and weight as well as shoot dry weight were recorded. Cantaloupe roots were stained according to the method of Byrd et al. (1983) and examined for the developmental stages, females and egg masses under stereomicroscope. Vermiform stages of $R$. reniformis were extracted from soil using sieving and modified Baermann technique (Goodey, 1957). Egg masses index (EI) were rated on a scale of 1-9 where $1=$ no egg masses; $2=1-5 ; 3=6-10 ; 4=11-15 ; 5=16-20 ; 6=21-30 ; 7=31-40 ; 8=41-50$ and $9=>50$ egg masses (Sharma and Ashakkumar, 1991).The obtained data were subjected to analysis of variance (ANOVA) (Gomez and Gomez, 1984), followed by Duncan's multiple range tests to compare means (Duncan, 1955). 


\section{Results and Discussion}

The influence of five alkaloid plants namely $C$. roseus, $D$. stramonium, $L$. albus, $P$. granatum and $T$. foenum-graecum at three tested rates (1, 3 and $5 \mathrm{~g} / \mathrm{plant})$ on plant growth response of cucumber infected with $M$. incognita is depicted in Table 1. The root knot nematode, $M$. incognita caused a significant reduction in plant growth parameters (shoot and root lengths, shoot weight) with reduction percentage in total plant fresh weight reached $26.3 \%$. Irrespective to tested rates, all treatments induced remarkable improvement in plant growth parameters with various degrees. Shoot length of cucumber infected with $M$. incognita was significantly $(P \leq 0.05)$ improved by all treatments of alkaloid plants where seed powder of $T$. foenum-graceum in par with dried leaf powder of $C$. roseus at three tested rates performed the best. It was evident that the effectiveness of dried leaf powder of $C$. roseus as well as seed powder of $L$. albus and $T$. foenum-graecum to enhance total plant fresh weight increased with the increase of tested rates with

Table (1): Impact of dried powder of five botanicals rich in alkaloids on growth parameters of cucumber (Cucumis sativus) infected with Meloidogyne incognita under greenhouse conditions at $22 \pm 5^{\circ} \mathrm{C}$.

\begin{tabular}{|c|c|c|c|c|c|c|c|c|c|}
\hline \multirow[b]{2}{*}{ Treatments } & \multirow[t]{2}{*}{ es (g) } & \multicolumn{2}{|c|}{$\begin{array}{l}\text { Length } \\
(\mathrm{cm})\end{array}$} & \multicolumn{2}{|c|}{$\begin{array}{c}\text { Weight } \\
\text { (g) }\end{array}$} & \multirow{2}{*}{$\begin{array}{c}\text { Total } \\
\text { fresh } \\
\text { weight } \\
\text { (g) }\end{array}$} & \multirow{2}{*}{ 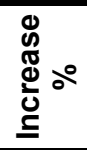 } & \multirow{2}{*}{$\begin{array}{l}\text { Shoot } \\
\text { dry } \\
\text { weight }\end{array}$} & \multirow{2}{*}{ 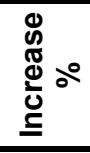 } \\
\hline & & Shoot & Root & Shoot & Root & & & & \\
\hline \multirow{3}{*}{$\begin{array}{l}\text { Catharanthus } \\
\text { roseus }\end{array}$} & 1 & $34.0^{\mathrm{bc}}$ & $9.16^{\mathrm{cd}}$ & $18.7^{\mathrm{d}}$ & $0.79^{b-f}$ & 19.49 & 33.8 & $2.67^{d}$ & 59.9 \\
\hline & 3 & $34.0^{b c}$ & $8.00^{c-e}$ & $21.6^{\mathrm{a}-\mathrm{c}}$ & $0.88^{a-c}$ & 22.48 & 54.4 & $2.80^{\mathrm{cd}}$ & 67.7 \\
\hline & 5 & $36.0^{\mathrm{ab}}$ & $8.00^{c-e}$ & $22.9^{a}$ & $0.76^{c-g}$ & 23.66 & 62.5 & $3.14^{\mathrm{b}}$ & 88.0 \\
\hline \multirow{4}{*}{$\begin{array}{c}\text { Datura } \\
\text { stramonium }\end{array}$} & 1 & $23.0^{\mathrm{hi}}$ & $7.50^{c-f}$ & $15.3^{\text {ef }}$ & $0.74^{e-g}$ & 16.04 & 10.2 & $1.98^{\mathrm{fg}}$ & 18.6 \\
\hline & 3 & $29.3^{\mathrm{d}-\mathrm{f}}$ & $9.66^{\mathrm{C}}$ & $20.0^{b-d}$ & $0.59^{\mathrm{h}}$ & 20.59 & 41.4 & $2.80^{\mathrm{cd}}$ & 67.7 \\
\hline & 5 & $25.0^{g-i}$ & $9.50^{c d}$ & $17.4^{\mathrm{de}}$ & $0.75^{\mathrm{d}-\mathrm{g}}$ & 18.15 & 24.6 & $2.30^{\mathrm{ef}}$ & 37.7 \\
\hline & 1 & $26.6^{\mathrm{f}-\mathrm{h}}$ & $12.30^{\mathrm{b}}$ & $17.3^{\mathrm{de}}$ & $0.88^{a-c}$ & 18 & 24.8 & $2.01^{\mathrm{fg}}$ & 20.4 \\
\hline \multirow[t]{3}{*}{ Lupinus albus } & 3 & $30.6^{\mathrm{c}-\mathrm{e}}$ & $16.00^{\mathrm{a}}$ & $18.5^{\mathrm{d}}$ & $0.97^{\mathrm{a}}$ & 19.47 & 33.7 & $2.01^{\mathrm{fg}}$ & 20.4 \\
\hline & 5 & $35.3^{\mathrm{ab}}$ & $9.50^{\mathrm{cd}}$ & $21.5^{\mathrm{a}-\mathrm{c}}$ & $0.75^{\mathrm{d}-\mathrm{g}}$ & 22.25 & 52.8 & $3.03^{\mathrm{bc}}$ & 81.4 \\
\hline & 1 & $27.3^{\mathrm{e}-\mathrm{g}}$ & $8.67^{c-e}$ & $15.3^{\mathrm{ef}}$ & $0.89^{\mathrm{ab}}$ & 16.19 & 11.2 & $1.98^{\mathrm{fg}}$ & 18.6 \\
\hline \multirow[t]{3}{*}{ Punica granatum } & 3 & $27.7^{\mathrm{e}-\mathrm{g}}$ & $6.30^{\mathrm{ef}}$ & $15.7^{\mathrm{ef}}$ & $0.67^{\mathrm{gh}}$ & 16.37 & 12.4 & $1.99^{\mathrm{fg}}$ & 19.2 \\
\hline & 5 & $29.6^{\mathrm{d}-\mathrm{f}}$ & $9.30^{\mathrm{cd}}$ & $17.7^{\mathrm{de}}$ & $0.90^{\mathrm{ab}}$ & 18.60 & 27.7 & $2.16^{f}$ & 29.3 \\
\hline & 1 & $34.3^{\mathrm{bc}}$ & $7.16^{\mathrm{d}-\mathrm{f}}$ & $19.4^{\mathrm{b}-\mathrm{d}}$ & $0.77^{\mathrm{b}-\mathrm{g}}$ & 20.17 & 38.5 & $3.21^{\mathrm{b}}$ & 92.2 \\
\hline \multirow{2}{*}{$\begin{array}{c}\text { Trigonella } \\
\text { foenum-graecum }\end{array}$} & 3 & $34.3^{\mathrm{bc}}$ & $6.50^{\mathrm{ef}}$ & $20.0^{b-d}$ & $0.71^{\mathrm{fg}}$ & 20.71 & 42.2 & $3.22^{b}$ & 92.8 \\
\hline & 5 & $39.0^{\mathrm{a}}$ & $5.50^{f}$ & $20.1^{\mathrm{b}-\mathrm{d}}$ & $0.75^{\mathrm{d}-\mathrm{g}}$ & 20.85 & 43.2 & $3.33^{\mathrm{b}}$ & 99.4 \\
\hline \multicolumn{2}{|l|}{ Oxamyl } & $33.0^{. b-d}$ & $8.66^{c-e}$ & $21.8^{\mathrm{ab}}$ & $0.95^{\mathrm{a}}$ & 22.75 & 56.3 & $3.68^{a}$ & 120.4 \\
\hline \multicolumn{2}{|l|}{ Nematode alone } & $21.6^{i}$ & $8.00^{c-e}$ & $13.7^{f}$ & $0.86^{a-e}$ & 14.56 & - & $1.67^{\mathrm{g}}$ & - \\
\hline \multicolumn{2}{|l|}{ Untreated plant } & $32.6^{b-d}$ & $12.00^{\mathrm{b}}$ & $18.9^{\text {cd }}$ & $0.87^{a-d}$ & 19.77 & 35.8 & $2.55^{\mathrm{de}}$ & 52.7 \\
\hline
\end{tabular}

Each value is the mean of three replicates.

Means in each column followed by the same letter(s) are not significantly different $(P \leq 0.05)$ by Duncan's multiple range test. 
percentage of increase reached $62.5,52.8$, and $43.2 \%$, respectively at higher rate (5g/plant). However, dried leaf powder of $D$. stramonium $(41.4 \%)$ achieved a significant increase in total plant fresh weight at the rate of $3 \mathrm{~g} /$ plant. Whereas at higher rate $(5 \mathrm{~g} /$ plant) of $D$. stramonium showed phytotoxic effects with reduction in growth parameters (Table1). Nevertheless, dried fruit peels of $P$. granatum at all tested rates showed moderately improvement in cucumber growth with percentages of increase in total plant fresh weight ranged from 11.2-27.7\%. On the other hand, the highest significant $(P \leq 0.05)$ improvement in shoot dry weight of cucumber was recorded with oxamyl (120.4\%) followed by dried seed powder of $T$. foenumgraecum $(99.4 \%)$ at the rate of $5 \mathrm{~g} /$ plant.

Results shown in Table (2) revealed that total nematode population was significantly suppressed by all tested treatments with reproduction factor (Rf) ranged from 0.42 to 0.94 . Among tested treatments, $C$. roseus performed the best and significantly suppressed total root-knot nematode population with percentage of reduction ranged from 71.6 to $72.6 \%$. Apparently, the higher the rate of alkaloid plants the greater suppression in nematode population was observed. Dried seed powder of $T$. foenum-graecum $(70.1 \%)$, dried leaf powder of $D$. stramonium $(69.4 \%)$, dried peels of $P$. granatum $(69.2 \%)$ and dried seed powder of $L$. albus $(69.0 \%)$ gave the greater suppression in nematode population whether in soil or roots at the rate of $5 \mathrm{~g} /$ plant than did other ones. Moreover, between treatments, pots receiving dried powder of alkaloid plants showed no significant differences $(P \leq 0.05)$ in nematode population in soil at higher rate $(5 \mathrm{~g} / \mathrm{plant})$. Root galling and number of egg masses were significantly reduced by all treatments with RGI ranged from 0.66 to 3.00 and El ranged from 0.0 to 3.0. Oxamyl as a standard nematicide was the superior and showed the highest reduction in total nematode population $(93.6 \%)$, number of galls $(97.3 \%)$ and egg masses $(100 \%)$.

The impact of dried powder of the previous five alkaloid plants at three tested rates ( 1,3 and $5 \mathrm{~g} /$ plant) on growth response of cantaloupe and $R$. reniformis population and reproduction is shown in Tables (3\&4). Among these plant species, dried leaf powder of $C$. roseus $(19.5 \%)$ as well as seed powder of $T$. foenumgraecum $(19.4 \%)$ and $L$. albus (13.9\%) at $3 \mathrm{~g} /$ plant showed detectable improvement in shoot and root length of cantaloupe compared to untreated inoculated plants.

A slight to moderate improvement in plant fresh weight was also noticed in all treatments, including oxamyl, with percentages of increase ranged from 9.3 to $32.6 \%$. The greatest increment in plant fresh weight was recorded with pots receiving dried leaf powder of $C$. roseus with values ranged from 28.5 to $32.6 \%$. Dried peels of $P$. granatum ranked the next with values ranged from 26.3 to $30.0 \%$ then dried seed of $L$. albus with values ranged from $13.4-16.8 \%$. On the other hand, oxamyl $(54.3 \%)$ exceeded other treatments and showed the highest percentage 
Table (2): Influence of dried powder of five botanicals rich in alkaloids on population and reproduction of Meloidogyne incognita infecting cucumber cv. Themin under greenhouse conditions at $22 \pm 5^{\circ} \mathrm{C}$.

\begin{tabular}{|c|c|c|c|c|c|c|c|c|c|c|c|}
\hline \multirow[b]{3}{*}{ Treatments } & & \multicolumn{3}{|c|}{ Nematode population in } & \multirow{3}{*}{ Total } & \multirow{3}{*}{$\mathbf{R f}^{*}$} & \multirow{3}{*}{$\begin{array}{c}\text { Red. } \\
\%\end{array}$} & \multirow{3}{*}{$\begin{array}{l}\text { No. } \\
\text { galls }\end{array}$} & \multirow{3}{*}{$\mathrm{RGI}^{* *}$} & \multirow{3}{*}{$\begin{array}{l}\text { No. egg } \\
\text { masses }\end{array}$} & \multirow{3}{*}{$\mathrm{El}^{* *}$} \\
\hline & & \multirow[b]{2}{*}{ Soil } & \multicolumn{2}{|c|}{ Root } & & & & & & & \\
\hline & & & $\begin{array}{c}\text { Develop- } \\
\text { mental } \\
\text { stages }\end{array}$ & Females & & & & & & & \\
\hline \multirow{4}{*}{ Catharanthus roseus } & 1 & $421^{d}$ & $5.66^{b}$ & $7.30^{f}$ & 433.96 & 0.43 & 71.8 & $12.0^{\mathrm{de}}$ & 2.7 & $6.0^{\mathrm{d}-\mathrm{f}}$ & 2.0 \\
\hline & 3 & $416^{d}$ & $10.30^{\mathrm{a}}$ & $10.67^{\mathrm{c}-\mathrm{e}}$ & 436.97 & 0.44 & 71.6 & $12.0^{\mathrm{de}}$ & 2.7 & $6.0^{\mathrm{d}-\mathrm{f}}$ & 2.0 \\
\hline & 5 & $410^{d}$ & $3.67^{\mathrm{cd}}$ & $8.30^{\text {ef }}$ & 421.97 & 0.42 & 72.6 & $9.3^{\mathrm{e}}$ & 2.0 & $5.7^{\text {ef }}$ & 2.0 \\
\hline & 1 & $571^{\mathrm{c}}$ & $3.00^{d-f}$ & $15.67^{b}$ & 589.67 & 0.59 & 61.7 & $16.3^{\mathrm{bc}}$ & 3.0 & $9.3^{\mathrm{cd}}$ & 2.3 \\
\hline \multirow[t]{3}{*}{ Datura stramonium } & 3 & $458^{d}$ & $2.67^{d-g}$ & $12.30^{\mathrm{c}}$ & 472.97 & 0.47 & 69.3 & $10.7^{\mathrm{de}}$ & 2.3 & $9.0^{c-e}$ & 2.3 \\
\hline & 5 & $459^{d}$ & $2.00^{e-g}$ & $9.67^{c-f}$ & 470.67 & 0.47 & 69.4 & $11.3^{\mathrm{de}}$ & 2.7 & $5.0^{f}$ & 2.0 \\
\hline & 1 & $626^{\mathrm{c}}$ & $2.30^{\mathrm{d}-\mathrm{g}}$ & $12.67^{\mathrm{c}}$ & 640.97 & 0.64 & 58.4 & $14.0^{\text {cd }}$ & 3.0 & $8.0^{c-f}$ & 2.3 \\
\hline \multirow[t]{3}{*}{ Lupinus albus } & 3 & $568^{c}$ & $3.66^{\mathrm{cd}}$ & $10.00^{c-f}$ & 581.66 & 0.58 & 62.2 & $11.7^{\text {de }}$ & 3.0 & $8.0^{c-f}$ & 2.0 \\
\hline & 5 & $463^{d}$ & $2.00^{e-g}$ & $12.30^{\mathrm{C}}$ & 477.30 & 0.48 & 69.0 & $11.7^{\mathrm{de}}$ & 3.0 & $8.0^{c-f}$ & 2.0 \\
\hline & 1 & $924^{\mathrm{b}}$ & $3.30^{\text {de }}$ & $17.66^{b}$ & 944.96 & 0.94 & 38.7 & $19.0^{\mathrm{b}}$ & 3.0 & $15.0^{b}$ & 3.0 \\
\hline \multirow[t]{2}{*}{ Punica granatum } & 3 & $620^{\mathrm{c}}$ & $4.66^{\mathrm{bc}}$ & $17.30^{\mathrm{b}}$ & 641.96 & 0.64 & 58.3 & $18.6^{b}$ & 3.0 & $14.7^{\mathrm{b}}$ & 3.0 \\
\hline & 5 & $464^{d}$ & $1.66^{\mathrm{fg}}$ & $9.00^{\text {def }}$ & 474.66 & 0.47 & 69.2 & $10.0^{\mathrm{e}}$ & 2.3 & $8.0^{c-f}$ & 2.0 \\
\hline \multirow{3}{*}{$\begin{array}{c}\text { Trigonella foenum- } \\
\text { graecum }\end{array}$} & 1 & $603^{c}$ & $2.66^{\mathrm{d}-\mathrm{g}}$ & $10.30^{c-f}$ & 615.96 & 0.62 & 60.0 & $12.0^{\mathrm{de}}$ & 3.0 & $10.0^{c}$ & 2.3 \\
\hline & 3 & $470^{d}$ & $2.66^{\mathrm{d}-\mathrm{g}}$ & $10.00^{c-f}$ & 482.66 & 0.48 & 68.7 & $12.3^{\text {de }}$ & 2.7 & $9.3^{c d}$ & 2.0 \\
\hline & 5 & $447^{d}$ & $1.33^{\mathrm{g}}$ & $11.66^{\mathrm{cd}}$ & 459.99 & 0.46 & 70.1 & $11.3^{\text {de }}$ & 2.7 & $8.3^{c-f}$ & 2.0 \\
\hline Oxamyl & & $97^{e}$ & $0.00^{\mathrm{h}}$ & $1.00^{g}$ & 98.00 & 0.09 & 93.6 & $1.0^{f}$ & 0.66 & $0.00^{9}$ & 0.0 \\
\hline Nematode alone & & $1502^{\mathrm{a}}$ & $5.30^{b}$ & $33.00^{\mathrm{a}}$ & 1540.3 & 1.54 & -- & $36.7^{\mathrm{a}}$ & 4.00 & $25.00^{\mathrm{a}}$ & 3.0 \\
\hline
\end{tabular}

${ }^{*}$ Reproduction factor $(\mathrm{Rf})=$ Final population / Initial population.

** Root gall index (RGI) or egg masses index (EI) scale: $0=$ no galls or egg masses, $1=1-2,2=$ $3-10,3=11-30,4=31-100$ and $5=$ more than 100 galls or egg masses $/$ root system. Means in each column followed by the same letter(s) are not significantly different $(P \leq 0.05)$ by Duncan's multiple range test. 
of increase in shoot dry weight, followed by dried seed of $T$. foenum-graecum $(44.6 \%)$ then dried seed of $L$. albus $(38.0 \%)$ and dried peels of $P$. granatum $(37.1 \%)$ at the higher rate (Table 3 ).

Table (3): Impact of five botanicals rich in alkaloids on growth parameters of cantaloupe (Cucumis melo var. cantalupensis) infected with Rotylenchulus reniformis under greenhouse conditions at $27 \pm 3^{\circ} \mathrm{C}$.

\begin{tabular}{|c|c|c|c|c|c|c|c|c|c|}
\hline \multirow{2}{*}{\multicolumn{2}{|c|}{ Rates (g) }} & \multicolumn{2}{|c|}{$\begin{array}{l}\text { Length } \\
(\mathrm{cm})\end{array}$} & \multicolumn{2}{|c|}{$\begin{array}{c}\text { Weight } \\
\text { (g) }\end{array}$} & \multirow{2}{*}{$\begin{array}{l}\text { Total } \\
\text { fresh } \\
\text { weight } \\
\text { (g) }\end{array}$} & \multirow{2}{*}{ 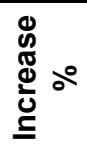 } & \multirow{2}{*}{$\begin{array}{c}\text { Shoot } \\
\text { dry } \\
\text { weight }\end{array}$} & \multirow{2}{*}{ 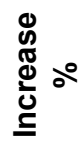 } \\
\hline & & Shoot & Root & hoot & Root & & & & \\
\hline \multirow{3}{*}{$\begin{array}{l}\text { Catharanthus } \\
\text { roseus }\end{array}$} & 1 & $\overline{12}$ & $\overline{0^{\mathrm{e}}}$ & $\mathrm{b}$ & ef & 51.89 & 28.5 & $\partial^{\mathrm{d}}$ & 25.7 \\
\hline & 3 & $125.0^{\mathrm{a}}$ & $28.3^{a-c}$ & $50.1^{\mathrm{ab}}$ & $2.96^{\mathrm{a}-\mathrm{c}}$ & 3.06 & 31.4 & & 27.4 \\
\hline & 5 & & $27.8^{\mathrm{a}-\mathrm{d}}$ & $51.4^{\mathrm{a}}$ & & 56 & 32.6 & & 31 \\
\hline \multirow{4}{*}{$\begin{array}{c}\text { Datura } \\
\text { stramonium }\end{array}$} & 1 & $100.3^{f}$ & $21.7^{\mathrm{e}}$ & $42.5^{\mathrm{de}}$ & $.59^{c-e}$ & 45.09 & 11.6 & & 0.0 \\
\hline & 3 & $103.0^{\text {ef }}$ & $21.3^{\mathrm{e}}$ & $43.2^{\mathrm{de}}$ & $2.03^{f}$ & 45.23 & 12.0 & $9^{e}$ & 2.6 \\
\hline & 5 & $107.0^{\mathrm{d}-\mathrm{f}}$ & 24.3 & $44.2^{\mathrm{de}}$ & -e & 46.82 & 15.9 & & 7.4 \\
\hline & & & $24.3^{\mathrm{a}-\mathrm{e}}$ & $43.9^{\mathrm{de}}$ & $1.94^{f}$ & 84 & 13.5 & & 24.6 \\
\hline \multirow[t]{3}{*}{ Lupinus albus } & 3 & $117.3^{\mathrm{a}-\mathrm{d}}$ & $28.7^{\mathrm{ab}}$ & $43.6^{\mathrm{de}}$ & $2.20^{\mathrm{ef}}$ & & 13.4 & & 32.6 \\
\hline & 5 & & $22.3^{\text {de }}$ & $3^{\text {de }}$ & $b c$ & 16 & 16.8 & & 38.0 \\
\hline & T & & $22.3^{\mathrm{de}}$ & $48.7^{\mathrm{a}-\mathrm{c}}$ & 2.3 & 0 & 26.3 & & 28. \\
\hline \multirow[t]{2}{*}{ Punica granatum } & 3 & & & & & & 27.3 & & 28.6 \\
\hline & 5 & 120 & $29.7^{\mathrm{a}}$ & $49.1^{\mathrm{a}-\mathrm{c}}$ & $3.40^{\mathrm{a}}$ & & 30.0 & & 37. \\
\hline \multirow{3}{*}{$\begin{array}{c}\text { Trigonella } \\
\text { foenum-graecum }\end{array}$} & 1 & $107.7^{c-f}$ & $23.7^{\mathrm{b}-\mathrm{e}}$ & $41.4^{\mathrm{ef}}$ & $2.76^{b-d}$ & 4 & 9.3 & & 33. \\
\hline & 3 & $120.0^{\mathrm{ab}}$ & $24.7^{\mathrm{a}-\mathrm{e}}$ & $42.2^{\mathrm{de}}$ & $3.00^{a-c}$ & 20 & 11.9 & a-c & 42. \\
\hline & 5 & $123.3^{\mathrm{a}}$ & $23.3^{\mathrm{b}-\mathrm{e}}$ & $42.9^{\mathrm{de}}$ & $3.40^{\mathrm{a}}$ & 46.30 & 14.6 & $6^{\mathrm{ab}}$ & 44. \\
\hline \multicolumn{2}{|l|}{ Oxamyl } & $112.3^{\mathrm{b}-\mathrm{e}}$ & $22.7^{\mathrm{ce}}$ & $45.1^{\mathrm{c}-\mathrm{e}}$ & $3.40^{\mathrm{a}}$ & 48.50 & 20.1 & $5.40^{\mathrm{a}}$ & 54.3 \\
\hline \multicolumn{2}{|l|}{ Nematode alone } & $107.7^{c-f}$ & $20.5^{\mathrm{e}}$ & $38.2^{f}$ & $2.19^{\mathrm{ef}}$ & 40.39 & -- & & \\
\hline \multicolumn{2}{|l|}{ Untreated plant } & $119.3^{\mathrm{ab}}$ & $25.7^{\mathrm{a}-\mathrm{e}}$ & $46.2^{b-d}$ & $3.26^{\mathrm{ab}}$ & 49.46 & 22.5 & $4.56^{\mathrm{b}-\mathrm{d}}$ & 30.3 \\
\hline
\end{tabular}

Each value is the mean of three replicates

Means in each column followed by the same letter(s) are not significantly different $(P \leq 0.05)$ by Duncan's multiple range test.

Apparently, R. reniformis population within soil as well as number of sessile females, observed on cantaloupe roots, were significantly $(P \leq 0.05)$ suppressed by all tested treatments with reproduction factor ranged from 0.06 to 0.97 (Table 4). Out of the five plants, dried seed powder of $T$. foenum-graecum $(74.6 \%)$ as well as dried peels of $P$. granatum $(61.7 \%)$ recorded the potent significant $(P \leq 0.05)$ reduction in total nematode population at higher rate $(5 \mathrm{~g} / \mathrm{plant})$ followed by dried seed powder of $L$. albus (58.5\%). Number of egg masses were significantly $(\mathrm{P} \leq 0.05)$ reduced with all pots receiving dried powder of alkaloid plants with egg masses index ranged from 3.0 to 5.0 . However, oxamyl $(96.8 \%)$ surpassed all treatments and significantly suppressed number of $R$. reniformis within soil and root $(\mathrm{Rf}=0.06)$ as well as number of egg masses $(E \mathrm{I}=1.0)$ compared to untreated inoculated plants $(E \mathrm{I}=8.0)$. 
Table (4): Influence of dried powder of five botanicals rich in alkaloids on population and reproduction of Rotylenchulus reniformis infecting cantaloupe under greenhouse conditions $27 \pm 3^{\circ} \mathrm{C}$.

\begin{tabular}{|c|c|c|c|c|c|c|c|c|}
\hline \multicolumn{2}{|c|}{$\mathrm{P}_{\text {Treatments }}$ Rates $(\mathbf{g})$} & \multirow{2}{*}{$\begin{array}{c}\begin{array}{c}\text { No. } \\
\text { nematodes } \\
\text { in soil }\end{array} \\
469^{\mathrm{b}}\end{array}$} & \multirow{2}{*}{$\begin{array}{c}\begin{array}{c}\text { No. females } \\
\text { in root }\end{array} \\
14.66^{\mathrm{c-f}}\end{array}$} & \multirow{2}{*}{$\begin{array}{c}\text { Total } \\
483.66\end{array}$} & \multirow{2}{*}{$\begin{array}{c}\mathbf{R f}^{*} \\
0.97\end{array}$} & \multirow{2}{*}{$\begin{array}{c}\begin{array}{c}\text { Red. } \\
\%\end{array} \\
48.2\end{array}$} & \multirow{2}{*}{$\begin{array}{c}\begin{array}{c}\text { No. egg } \\
\text { masses }\end{array} \\
13.3^{\mathrm{b}-\mathrm{d}}\end{array}$} & \multirow{2}{*}{$\frac{\mathrm{El}^{\star *}}{4.0}$} \\
\hline \multirow{3}{*}{$\begin{array}{l}\text { Catharanthus } \\
\text { roseus }\end{array}$} & 1 & & & & & & & \\
\hline & 3 & $400^{b-e}$ & $17.00^{\mathrm{cd}}$ & 417.00 & 0.83 & 55.3 & $13.0^{b-d}$ & 4.0 \\
\hline & 5 & $387^{\mathrm{c}-\mathrm{e}}$ & $10.00^{\text {ef }}$ & 397.00 & 0.79 & 57.5 & $9.0^{\mathrm{de}}$ & 3.0 \\
\hline \multirow{3}{*}{$\begin{array}{c}\text { Datura } \\
\text { stramonium }\end{array}$} & 1 & $462^{b}$ & $19.30^{\mathrm{bc}}$ & 481.30 & 0.96 & 48.5 & $18.7^{\mathrm{b}}$ & 5.0 \\
\hline & 3 & $460^{b}$ & $14.00^{c-f}$ & 474.00 & 0.95 & 49.2 & $11.0^{\mathrm{de}}$ & 4.0 \\
\hline & 5 & $440^{b-d}$ & $16.30^{\mathrm{cd}}$ & 456.30 & 0.91 & 51.1 & $11.0^{\mathrm{de}}$ & 4.0 \\
\hline \multirow{3}{*}{ Lupinus albus } & 1 & $447^{\mathrm{bc}}$ & $15.00^{\mathrm{c}-\mathrm{e}}$ & 462.00 & 0.92 & 50.5 & $13.3^{\mathrm{b}-\mathrm{d}}$ & 4.0 \\
\hline & 3 & $376^{c-e}$ & $14.30^{c-f}$ & 390.00 & 0.78 & 58.2 & $11.7^{\mathrm{c}-\mathrm{e}}$ & 4.0 \\
\hline & 5 & $370^{d-f}$ & $17.30^{\mathrm{cd}}$ & 387.30 & 0.77 & 58.5 & $10.7^{\mathrm{de}}$ & 4.0 \\
\hline \multirow{3}{*}{ Punica granatum } & 1 & $405^{b-e}$ & $11.30^{\mathrm{d}-\mathrm{f}}$ & 416.30 & 0.83 & 55.4 & $8.7^{\mathrm{de}}$ & 3.0 \\
\hline & 3 & $398^{\mathrm{b}-\mathrm{e}}$ & $13.66^{\mathrm{c}-\mathrm{f}}$ & 41 & 0.82 & 55.9 & $8.7^{\text {de }}$ & 3.0 \\
\hline & 5 & $349^{\text {ef }}$ & $8.66^{f}$ & 357.66 & 0.72 & 61.7 & $6.7^{\mathrm{e}}$ & 3.0 \\
\hline \multirow{3}{*}{$\begin{array}{c}\text { Trigonella } \\
\text { foenum-graecum }\end{array}$} & 1 & $370^{d-f}$ & $23.30^{b}$ & 393.30 & 0.78 & 57.9 & $17.0^{\mathrm{bc}}$ & 5.0 \\
\hline & 3 & $306^{f}$ & $19.30^{\mathrm{bc}}$ & 325.30 & 0.65 & 65.2 & $11.0^{\mathrm{de}}$ & 4.0 \\
\hline & 5 & $218^{9}$ & $15.00^{\mathrm{c}-\mathrm{e}}$ & 233.00 & 0.47 & 75.0 & $9.7^{\mathrm{de}}$ & 3.0 \\
\hline \multicolumn{2}{|l|}{ Oxamyl } & $30^{\mathrm{h}}$ & $0.00^{9}$ & 30.00 & 0.06 & 96.8 & $0.0^{f}$ & 1.0 \\
\hline \multicolumn{2}{|l|}{ Nematode alone } & $877^{\mathrm{a}}$ & $56.66^{a}$ & 933.66 & 1.77 & -- & $42.0^{a}$ & 8.0 \\
\hline
\end{tabular}

${ }^{*}$ Reproduction factor (Rf) = Final population / Initial population

**Egg masses index (EI) was rated on a scale of $1-9$ where $1=$ no egg masses; $2=1-5 ; 3=6-10 ; 4=11-$ $15 ; 5=16-20 ; 6=21-30 ; 7=31-40 ; 8=41-50$ and $9=>50$.

Means in each column followed by the same letter(s) are not significantly different $(P \leq 0.05)$ by Duncan's multiple range test.

In the present investigation, irrespective to tested rates such botanicals containing alkaloids showed remarkable improvement in plant criteria of cucumber infected with $M$. incognita with different percentages of increase over control. However, in most treatments higher rates showed better results than did the other ones. The highest percentage of increase in total plant fresh weight was recorded with C. roseus $(62.5 \%)$, L. albus $(52.8 \%)$ and T. foenum-graecum $(43.2 \%)$ at $5 \mathrm{~g} /$ plant. In addition, dried leaf powder of $C$. roseus exerted a noteworthy nematicidal properities against $M$. incognita and significantly suppressed nematode population, gall formation and egg masses numbers at three tested concentrations. This result agreed with that reported by Ranjana (2015) in respect to the potential of the leaf extract of periwinkle against $M$. incognita infesting papaya. In previous studies, chemical analysis reveal the presence of alkaloids, saponins, flavanoids, carbohydrates and anthraquinone glycosidesinperiwinkle, C. roseus (Phani Deepthi Yadav et al., 2013). However, more than 100 alkaloids with variable 
proportions have so far been isolated and characterized from all parts of $C$. roseus (Barrales-Cureño, 2015). Periwinkle or $C$. roseus has been extensively studied because of its production of two valuable alkaloids, vincristine and vinblastine, which are responsible for anticancerous activity. Biologically indole alkaloids produced by plants are believed to exert antimicrobial and antifeeding properties (Chockalingam et al.,1989 and Luijendijk, et al.,1996).

At higher rate,dried seed powder of $T$. foenum-graecum $(70.1 \%)$ induced a substantial suppression in root-knot nematode, $M$. incognita population and reproduction which confirm the findings of Ibrahim et al. (2007). However, Patil and Jain (2014) reported the presence of bioactive compounds isolated from fenugreek seeds viz. saponins (fenugreekine, diosgenin), alkaloids (trigonelline, gentianine, carpaine), amino acids (4-Hydroxyisoleucin, arginine) and flavanoids. It is well known that trigonelline plays an important role in plant resistance against several pathogens (Tyihak et al., 1978). Trigonelline, the principle alkaloid found in fenugreek showed robust antibacterial (Suhad et al., 2004 and Ozçelik et al., 2011) and antiviral activities ( Bano et al., 2016). Researchers have postulated the inhibitory activity of trigonelline against enzyme acetylcholinesterase (Satheeshkumaret al., 2010) an enzyme that acts on termination of nerve impulses.

Dried leaf powder of $D$. stramonium applied at the rate of $3 \mathrm{~g} /$ plant induced a remarkable increment in total plant fresh weight $(41.4 \%)$ and significantly suppressed total root-knot nematode population (69.4\%) within soil and root. This result agreed with the findings of Umar and Ngwamdai (2015) who mentioned that sweet melon treated with the leaf powder of $D$. stramonium gave longer vine length, higher fruit weights, lowest galling index and fewer nematodes than the control plants. Researchers (Adegbite and Adesiyan, 2005 and Umar, 2012) suggested that the effect of the different rates of $D$. stramonium on juvenile mortality may be due to the presence of alkaloids and other chemical compounds which have been reported to kill nematodes. Also, Shahwar et al. (1995) stated that $D$. fastuosa has nematicidal activity which may be due to the presence of alkaloids compounds i.e. tigloidine, apoatropine, hyoscyamine and scopolamine. Phytochemical analysis by Alexander et al. (2008) indicated the presence of thirty different tropane alkaloids in D. stramonium. Of such alkaloids, hyoscyamine and scopolamine are predominamt in flower and leaves (Witte, et al. 1987). As reported by Muhammad (1996) scopolamine isolated from $D$. fastuosa showed significant nematicidal activity against M. javanica. The alkaloids killed 90 to $100 \%$ of Hoplolaimus indicus, Helicotylenchus multicinctus, and M. incognita (Qamar et al., 1995).

At higher concentration, dried fruit peels of $P$. granatum $(69.2 \%)$ in par with dried seed powder of $L$. albu $s(69.0 \%)$ showed significant suppression in nematode population. These results are in accordance with those reported by Ibrahim et al. (2007) who documented that soil treated with lupin, fenugreek, acacia seed 
powders and camphor dried leaves caused great reduction in root galling and egg masses numbers of $M$. incognita as well as resulted in a significant increase in growth parameters of sunflower plants. Chemical analysis of $L$. albus seeds showed the presence of novel lupin alkaloids i.e. termisine and lupanine (Mohamed and El-Shorbagi, 1993) and contain high amount of quinolizidine alkaloids (Santiago Quiles et al., 2010). El-Shazly et al. (2001) identified 29 compounds of quinolizidine, bipiperidyl and proto-indole alkaloids in L. termis seeds. The alkaloid seeds exhibited substantial activity against Candida albicans, Aspergillus flavus and B.subtilis.

However, chemical analysis of $P$. granatum revealed the presence of alkaloids in the root and body rinds (Mohammad and Kashani, 2012) and aqueous and ethanol extracts of fruit peel (Sangeetha and Jayaprakash, 2015). Eight types of ingredients were observed in the fruit peel pomegranate and only 2-p-nitrophenyl 1,3,4-oxadiazol-5 was found for alkaloids (Jung, 2015). Whilst and Prakash (2011) recorded the presence of alkaloid pelletierine (punicine) in pomegranate peel that was documented to have anthelminthic properties (Wibaut and Hollstein, 1956) and effective against tape worms, ring worms and nematodes (EI Sakka, 2010).

Apparently, a slight to moderate improvement in total plant fresh weight of cantaloupe infected with $R$.reniformis was recorded in pots receiving different rates of alkaloid plants. Dried fruit peels of $P$. granatum showed remarkable improvement in total plant fresh weight at three tested rates. Mashed fruit of $P$. granatum was found to improve shoot length and weight and weight of sugar beet roots in respect to $M$. incognita compared to nematode alone (El-Nagdi and Youssef, 2015). However, Ashraf and Khan (2008) reported that treatment with pomegranate did not significantly improved the plant growth of chickpea as compared to plants inoculated with $R$. reniformis alone. A significant reduction in nematode population was acheived by dried seed powder of $T$. foenum-graecum and dried fruit peels of $P$. granatum at three rates as well.

In current investigation the potential of selective alkaloid plants to suppress root-knot nematode and reniform nematode development and reproduction might be attributed to the presence of secondary metabolites in alkaloid botanicals i.e.vincristine and vinblastine (periwinkle), termisine and lupanine(lupin), trigonelline (fenugreek), tropane alkaloids (datura) and pelletierine (pomegranate). Owing to this property, phytochemicals isolation and identification of secondary metabolites of alkaloid plants are needed for further studies under laboratory, greenhouse and field conditions to exploit them for their possible application for the preservation of environment, plant and humans from nematicidal hazards. 


\section{References}

Adegbite, A. A. (2003). Comparative effects of carbofuran and water extract of Chromolaena odorata on growth, yield and food components of root-knot nematode-infested soybean (Glycine max (L.) Merrill). Ph.D. thesis, University of Ibadan, Ibadan, Nigeria.217 pp.

Adegbite, A.A and Adesiyan, S. O (2005). Root extracts of plants to control root knot nematodes on edible soyabeans. World J. Agric. Sci. 1(1):18-21

Alexander, J.; Benford, D.; Cockburn, A. (2008). Tropane alkaloids (from Datura sp.) as undesirable substances in animal feed. European Food Safety Authority, 691: 1-55.

Amar, Z. and Lev, E. (2011). Watermelon, chate melon, and cucumber: new light on traditional and innovative field crops in the Middle Ages. J. Asiatique. 299:193-204.

Archana U. S. and Prasad, D. (2014). Management of plant-parasitic nematodes by the use of botanicals. J. Plant Physiol. Pathol. 2(1):2-10.

Ashraf, M. S. and Khan, T. A. (2008). Biomanagement of reniform nematode, Rotylenchulus reniformis by fruit wastes and Paecilomyces lilacinus on chickpea. World J. Agricultural Sciences 4 (4): 492-494.

Bano, D.; Tabassum, H.; Ahmad, A.; Mahbood, A. and Ahmad. I. Z. (2016). The medicinal significance of bioactive compounds of Trigonella foenumgraecum: A review. Int. J. R. Ayurveda Pharm.,7(4):84-91. Doi:10.7897/ 2277- 4343.074139

Barrales-Cureño, H. J. (2015). Pharmacological applications and in vitro biotechnological production of anticancer alkaloids of Catharanthus roseus. Biotecnología Aplicada, 32(1):1101-1110.

Byrd, D. W.; Kirapatrick, T. and Barker, K. (1983). An improved technique for clearing and staining plant tissues for detection of nematodes. J. Nematol. 15(3)142-3.

Chockalingam, S.;NalinaSundari, M. S. and Thenmozhi, S. (1989). Impact of the extract of Catharanthus roseus on feeding and enzymatic digestive activities of Spodoptera litura. J. Environ. Biol., 10:303-307.

Dorman, M. and Nelson, S. (2012). Root-Knot Nematodes on Cucurbits in Hawai'i. Plant Disease, 84: 1-5.

Duncan, D. B. (1955). Multiple range and multiple F. test. Biometrics 11, 1-42.

El-Sazley, A., Ateya, A.M. and Wink, M.(2001). Quinolizidine alkaloid profiles of Lupinus variusorientalis, $L$. albus albus, $L$. hartwegii and $L$. densiflorus. $Z$. Naturforsch, 56c,21-30. 
El-Nagdi, W. M. A. and Youssef, M. M. A. (2015). Nematicidal effect of some aqueous extracts of botanicals and a commercial bacterial byproduct for biocontrolling root knot nematode, Meloidogyne incognita infecting sugar beet. Sci. Agric., 10 (2): 55-58.

El-Sakka M. A. (2010). Phytochemistry alkaloids. $3^{\text {rd }}$ edition, pp.7-22.

Gomez, K. A. and A. A. Gomez, (1984). Statistical Procedures for Agriculture Research.2nd Ed., June Wiley \& Sons. Inc. New York.

Goodey, J. B. (1957). Laboratory methods for work with plant and soil nematodes. Tech. Bull. No.2 Min. Agric. Fish Ed. London pp.47.

Goswami, B. K. and Vijaylakshmi, K. (1986). Nematicidal properties of some indigenous plant materials against root knot nematode Meloidogyne incognita on tomato. Indian J. Nematol., 16(1): 65-68.

Ibrahim, I. K. A.; EL-Saedy, M. A. M. and Mokbel, A. A. (2007). Control of the root-knot nematode Meloidogyne incognita on sunflower plants with certain organic plant materials and biocontrol agents. Egypt. J. Phytopathol.,35 (1): 13-24.

Ismail, A. E. (2014). Management of root-knot nematode Meloidogyne javanica on tomato by dry crushed pomegranate Punica granatum L. peels as a biofumigant in Egypt. Archives of Phytopathol. and Plant Protec., 48(3): 253258.

Jung, J. S. (2015). Insecticidal effects from ethanol extracts of root peel, stem peel and fruit peel of pomegranate (Punica granatum L.) on house dust mite International Journal of Bio-Science and Bio-Technology, 7 (2) :25-36.

Luijendijk, T. J. C.; Van der, M. E. and Verpoorte, R. (1996). Involvement of strictosidine as a defensive chemical in Catharanthus roseus. J Chem. Ecol., 22:1355-1366.

Mohamed, M. H. and El-Shorbagi, A. N. H. (1993). Termisine, a novel lupine alkaloid from the seeds of Lupinus termis. J. Nat. Prod., 56 (11): 19992002.

Mohammad, S. M. and Kashani, H. H. (2012). Chemical composition of the plant Punica granatum L. (pomegranate) and its effect on heart and cancer. J. Med. Plants Res., 6(40): 5306-5310.

Muhammad, A. (1996). Studies on the control of root-knot nematodes (Meloidogyne spp.) with botanical toxicants. https://www.researchgate.net/ publication/ 28347840.

Noling, J. W. (2009). Nematode management in cucurbits (cucumbers, melons, squash). http://edis.ifas.ufl.edu/ ng025. 
Ozçelik, B., Kartal, M. and Orhan, I. (2011). Cytotoxicity, antiviral and antimicrobial activities of alkaloids, flavonoids, and phenolic acids. Pharm. Biol., 49(4):396-402. doi: 10.3109/13880209.2010.519390

Patil, S. and Jain, G. (2014). Holistic approach of Trigonella foenum-graecum in Phytochemistry and Pharmacology- A Review.Current Trends in Technology and Science. 3(1):34-48.

Phani Deepthi Yadav, CH. S. D.; Bharadwaj, N. S. P.; Yedukondalu, M.; Methushala, CH. and Ravi Kumar, A. (2013). Phytochemical evaluation of Nyctanthes arbortristis, Nerium oleander and Catharunthus roseus. Indian J. Research in Pharmacy and Biotechnol., 1(3):333-338.

Prakash, C.V.S. and Prakash, I. (2011). Bioactive chemical constituents from pomegranate (Punica granatum) juice, seed and peel-A review. International Journal of Research in Chemistry and Environment, 1 (1): 118.

Qamar, F.; Kapadia, Z.; Khan, S. A. and Badar, Y. (1995). Datura metel L., a plant with nematicidal potential. Pak. J. Sci. Ind. Res., 38: 319-321.

Ranjana, L. S. (2015). Nematicidal activity of the leaf extract of Vinca rosea, carbofuran and their combinations against Meloidogyne incognita infesting papaya. Pesticide Research J. 27(2): 266- 270.

Sangeetha, R and Jayaprakash, A. (2015). Phytochemical screening of Punica granatum Linn. peel extracts. J. Academia and Industrial Research. 4(5):160-162.

Santiago Quiles, M. R., Oquendo-Jiménez, I.; Herreño-Saénz1, D. and Antoun, M. D . (2010). Genotoxicity of alkaloid-rich extract from Lupinus termis seeds. Pharmaceutical Crops.(1): 18-23.

Satheeshkumar N.; Mukherjee, P. K.; Bhadra S. and Saha B.P. (2009). Acetylcholinesterase enzyme inhibitory potential of standardized extract of Trigonella foenum-graecum $\mathrm{L}$ and its constituents. Phytomedicine,7(34):292-5.

Sharma, S. B. and Ashokkumar, P. (1991). A technique to evaluate pigeonpea for resistance to Rotylenchulus reniformis. Ann. Appl. Biol. 119:323-330.

Shahwar, D.; Abid M.; Rehman, A. U.; Maqbool, M. A. and Choudhary, M. I. (1995). Nematicidal compounds from Datura fastuosa. In: Proc. 19th IUPAC Symposium on the chemistry of Natural Products. Pp. 171-179. Atta-urRehman, Choudhary MA and Sheikhani MS (Eds.).HEJ Res. Inst. of Chemistry, University of Karachi, Karachi 75270- Pakistan. pp. 409.

Suhad, A. S. and Ekbal, A. and Ali A. (2004). Anti-microbial investigation of Suaeda baccata (chenopodiaceae). American J. Political Sci., 2(1): 49-51. 
Taylor, A. L. and Sasser, J. N. (1978). Biology, identification and control of rootknot nematodes (Meloidogyne spp.) Coop. Pub. Dept. Plant Pathol. North Carolina State Univ. and U.S. Agency Int. Dev. Raleigh, N.C. 111 pp.

Tyihak, E., Sarhan, A. R. T., Cong, N. T., Barna, B. and Kiraly, Z. (1978). The level of trigonelline and other quaternary ammonium compounds in tomato leaves in ratio to the changing nitrogen supply. Plant and Soil, 109:285-7. doi: 10.1007/BF02202097.

Umar, I. (2012). Comparative efficacy of four organic amendments for controlling root knot nematode in cowpea (Vigna unguiculata L.). Walp. J. Agric. Biotechnol. and Ecol., 5: 75-83.

Umar, I. and Ngwamdai, P. A. (2015). Evaluation of leaf powder and extract of Datura stramonium in controlling root knot nematode [Meloidogyne javanica (Chitwood) 1949] on sweet melon in Yola, Adamawa State. International J. Chemical, Environmen. \& Biological Sciences, 3(1) :96-100.

Wibaut, J. P. and Hollstein, U. (1956). Investigation of the alkaloids of Punica granatum L., Archive of Biochemistry and Biophysics, 69: 27-32.

Witte, L.; Muller, K. and Arfmann, H. A. (1987). Investigation of the alkaloid pattern of Datura innoxia plants by capillary gasliquid-chromatographymass-spectrometry. Planta Medica 53, 192-197.

Zia, T.; Siddiqui, I. A. and Hasnain, N. (2001). Nematicidal activity of Trigonella foenum-graecum L. Phytother. Res., 15: 538-540. 


\section{الملخص العربي}

إمكانية استخدام النباتات الغنية بمجموعة القلويدات فى مكافحة نيماتودا تعقد الجذور والنيماتودا

$$
\text { الكلوية التي تصيب القرعيات }
$$

فاطمة عبد المحسن مصطفى*، عبد الفتاح رجب رفاعي*، أشرف السعيد خليل **، ومروة محمود الدرينى*ثي

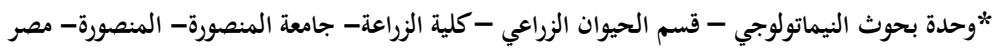

$$
\text { * * معهد بحوث أمراض النبات - مركز البحوث الزراعية - الجيزة - مصر. }
$$

تم دراسة تأثير مسحوق الأوراق الجافـة لنباتـات الونكا والـداتورة ومسحوق البذور لنباتـات

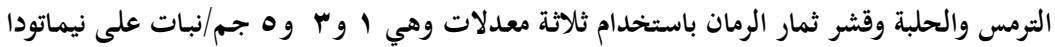

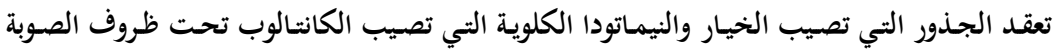

$$
\text { وأسفرت النتائج لنبات الخيار عن الآتي: }
$$

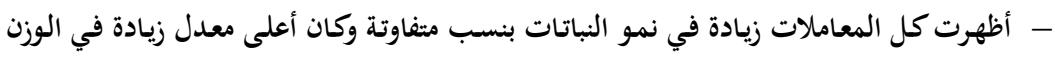

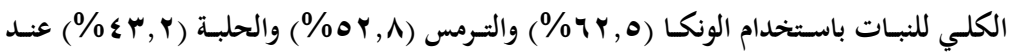

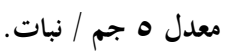

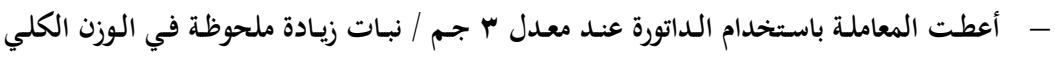

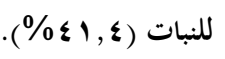

- أعطت المعاملة باستخدام الونكا عند المعدلات الثلاثة المستخدمة أعلى معدل خفض في تعداد

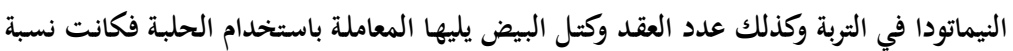

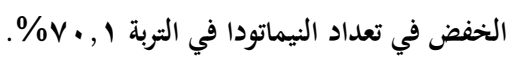

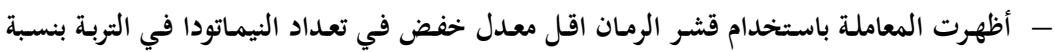

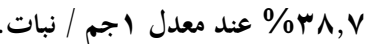

$$
\begin{aligned}
& \text { بالنسبة لنبات الكانتالوب: }
\end{aligned}
$$

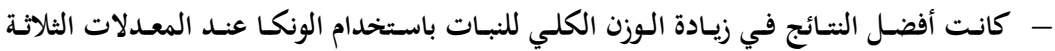

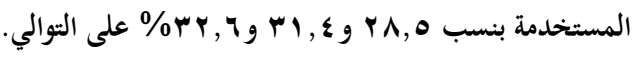

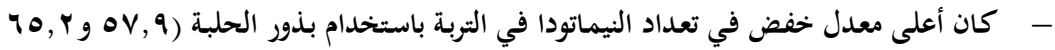

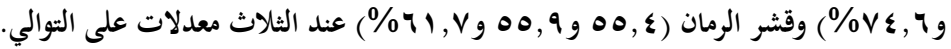

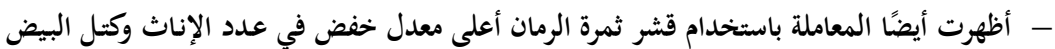

عند المعدلات الثلاثة المستخدمة.

- يمكن تفسير التأثير الأبادي للنباتات المختبرة إلى وجود المواد الفعالة التالية مثل:

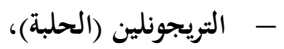

(الونكا)، (المان) vincristine vinblastine -

Pelletierine -

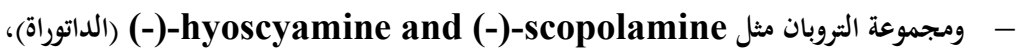

termisine and lupanine - 